\title{
Steep rise in notified hantavirus infections in Germany, April 2010
}

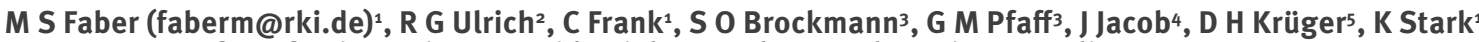

1. Department for Infectious Disease Epidemiology, Robert Koch Institute, Berlin, Germany

2. Friedrich-Loeffler Institute, Institute for Novel and Emerging Infectious Diseases, Greifswald - Insel Riems, Germany

3. State Health Office Baden-Württemberg, Stuttgart, Germany

4. Julius Kühn Institute, Federal Research Centre for Cultivated Plants, Institute for Plant Protection in Horticulture and Forestry, Vertebrate Research, Münster, Germany

5. Institute of Virology, Helmut-Ruska-Haus, Charité - Universitätsmedizin Berlin, National Consultation Laboratory for Hantaviruses, Berlin, Germany

Citation style for this article: Faber MS, Ulrich RG, Frank C, Brockmann SO, Pfaff GM, Jacob J, Krüger DH, Stark K. Steep rise in notified hantavirus infections in Germany, April 2010. Euro Surveill. 2010;15(20):pii=19574. Available online: http://www.eurosurveillance.org/ViewArticle.aspx?Articleld=19574

This article has been published on 20 May 2010

From January to April 2010, 396 hantavirus infections were notified in Germany, a considerable increase compared with previous years (mean: 83 for January-April 2004-2009) including the record-setting year, 2007 $(n=232$ January-April). Most patients are residents of known Puumala virus endemic areas in southern Germany. The recent increase in notified hantavirus infections is probably due to an increased population density of the main animal reservoir, the bank vole (Myodes glareolus).

\section{Introduction}

European hantaviruses of the family Bunyaviridae cause haemorrhagic fever with renal syndrome. Infection in humans occurs through inhalation of aerosolised virus particles from excreta of chronically infected wild rodents or, rarely, through rodent bites. Infection with Puumala virus - the hantavirus virus species most prevalent in northern and central Europe including Germany - leads to a relatively mild form of disease referred to as nephropathia epidemica. After an incubation period of 5-60 days, patients typically present with abrupt onset of fever and influenza-like symptoms followed by gastrointestinal symptoms. Acute kidney failure requiring temporary haemodialysis may develop.

Puumala virus epidemics in humans occur regularly in several European countries, particularly those in Fennoscandia, and have been linked to cyclic oscillations in the population density of the main animal reservoir, the bank vole (Myodes glareolus) [1]. In Germany, typically 150-250 cases have been notified annually since 2001. In 2005 and 2007, however, the annual number of cases peaked at 447 and 1,688, respectively. The outbreak in 2005 mainly affected the federal states of North Rhine-Westphalia and Lower Saxony, including increased numbers of human cases in urban areas [2,3], whereas in 2007 , when a record number of hantavirus cases were recorded, most of the cases were reported from the federal states of Baden-Württemberg and Bavaria $[4,5]$.

\section{Methods}

Laboratory-confirmed hantavirus infections have been notifiable in Germany since 2001. Serological evidence or detection of viral RNA by reverse transcriptionpolymerase chain reaction (RT-PCR) is reported to the local public health department by the identifying laboratory. The health department completes and verifies case information according to the national case definition [4]. Information about clinical signs and outcome is obtained either from the patients or their physicians. Case data are anonymised and electronically transmitted to the state health department and from there to the Robert Koch Institute, the national public health institute. For quality assurance, the information in each case report is checked at the Robert Koch Institute for compliance with the case definition and for data consistency. Early transmission of case data based purely on laboratory diagnosis is encouraged: it may take a few days or a few weeks to gather all the information relevant to the case definition and to complete the quality assurance process.

This report describes laboratory-confirmed hantavirus infections with clinical symptoms (according to the national case definition) reported during January to April 2010 for which quality assurance was completed, as of 14 May 2010. An additional 21 notifications in April 2010 currently undergoing quality assurance are not included in this report.

\section{Results}

The number of hantavirus cases notified in Germany rose continuously between November $2009(n=26)$ and March $2010(n=69)$, with a further steep increase in April $2010(n=166)$ (Figure 1). As case confirmation is pending for a further 21 notifications in April 2010, the number of cases in April is expected to rise. 
From January to April 2010, a total of 396 cases were notified (cumulative incidence: 0.5 per 100,000 population), compared with 13 cases in January - April 2009 and 232 in January - April 2007, the year with the highest number of notified infections so far.
The most common symptoms in cases notified in January to April 2010 were fever, renal impairment, muscle pain and headache (notified for $84 \%, 74 \%, 55 \%$ and $47 \%$ of cases, respectively). Of these, $64 \%$ were notified as having been hospitalised. The frequency of

\section{FIGURE 1}

Notified hantavirus infections by year and month of notification, Germany, January 2004 - April 2010 (n=3,269)

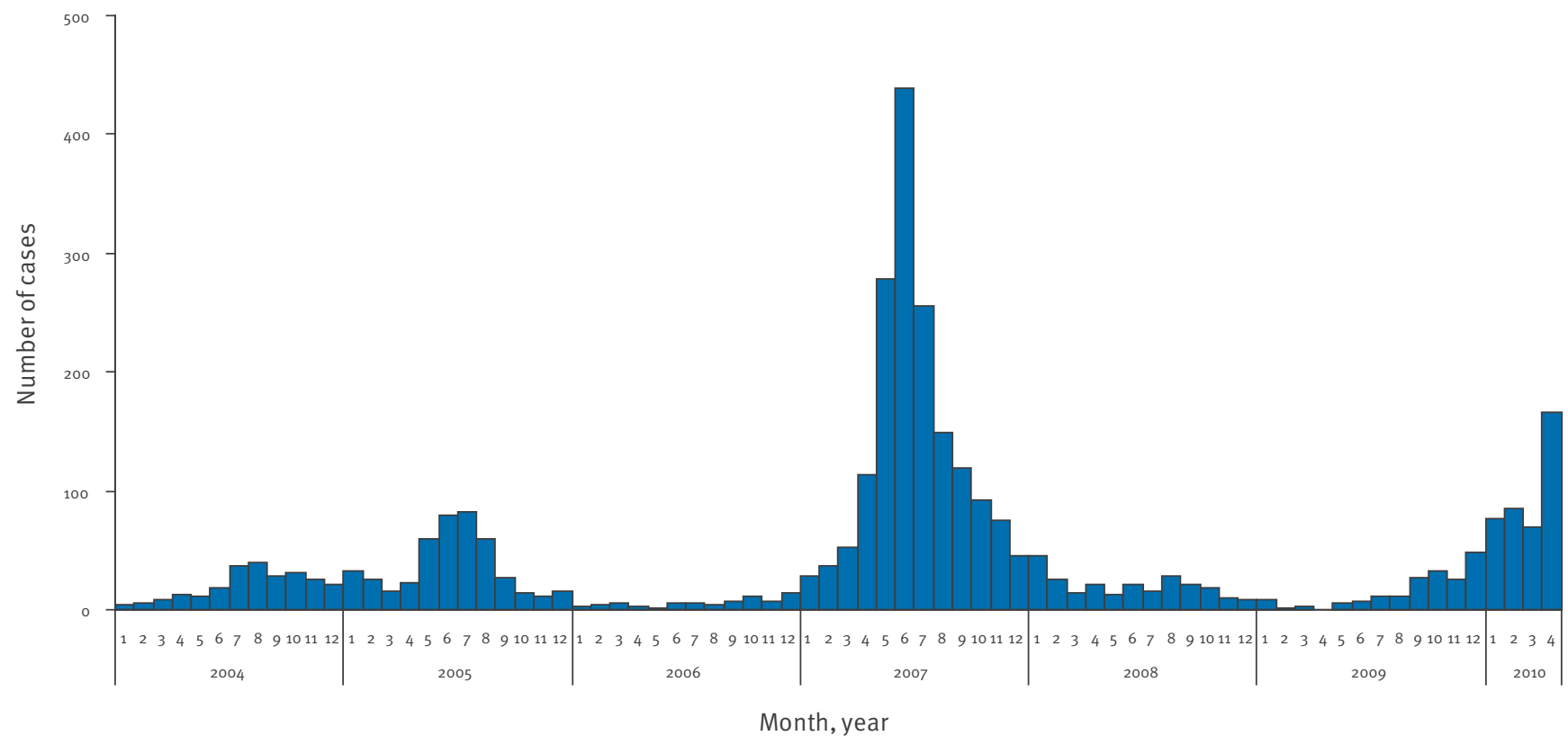

\section{FIGURE 2}

Cumulative incidence of notified hantavirus cases by age group and year, Germany, January - April 2007 ( $\mathrm{n}=232$ ) and January - April $2010(\mathrm{n}=396)$

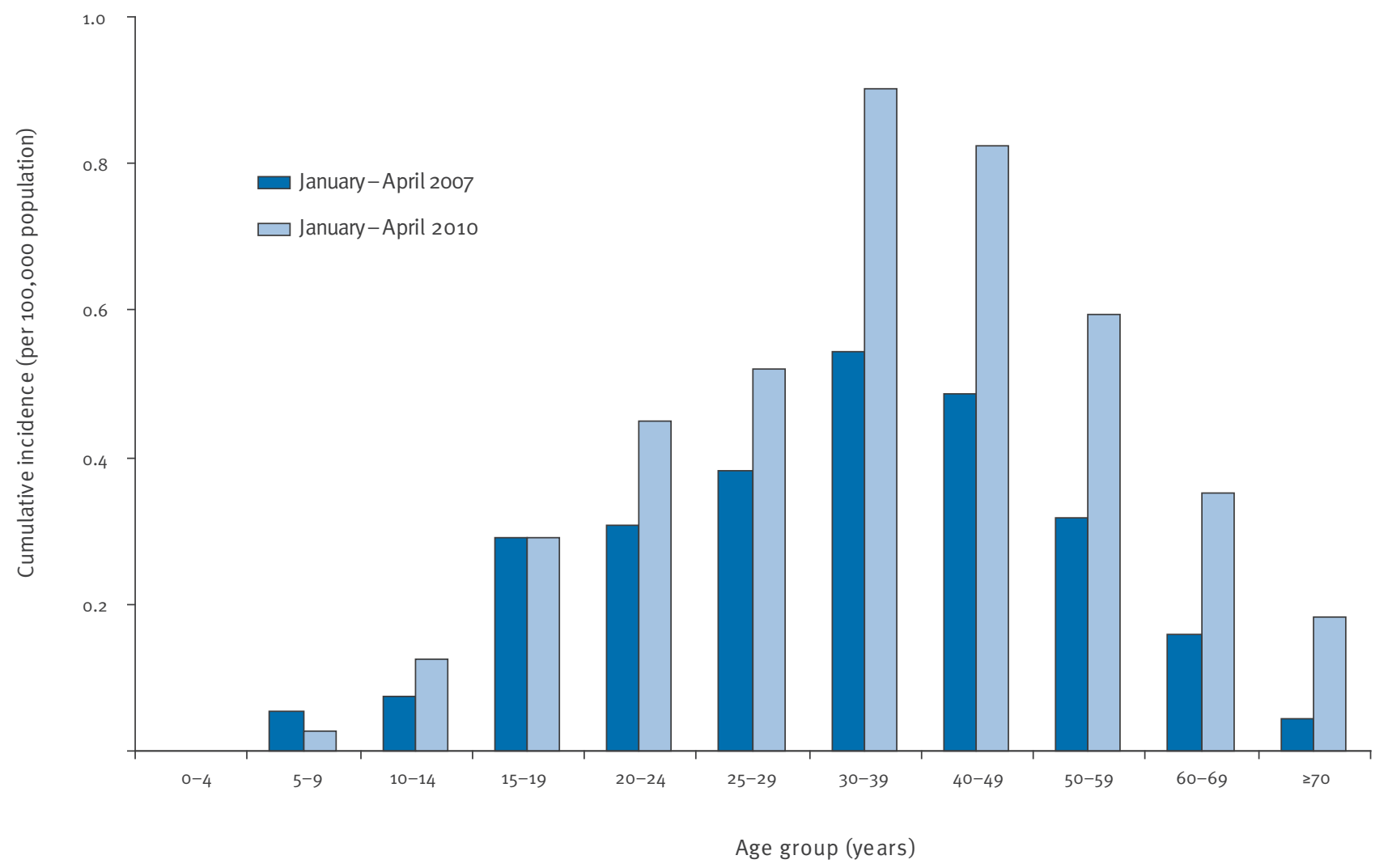


notified symptoms and the hospitalisation rate were comparable with those of previous years.

Of the 396 patients, 288 (72\%) were male and 275 $(69 \%)$ were between 30 and 59 years-old. Only one infection occurred in a person younger than 10 years. Compared with the first four months of 2007, the highest increase in incidence was observed in people older than 30 years (Figure 2).

Most hantavirus infections in January to April 2010 (78\%) were notified from two states in southern Germany, Baden-Württemberg (64\%) and Bavaria (14\%). A further $19 \%$ were notified from the western states of North Rhine-Westphalia, Hesse and Lower Saxony (7.8\%, 5.8\% and 5.8\%, respectively). No substantial increase of cases was observed in the federal states of Schleswig-Holstein and MecklenburgWestern Pomerania (Figure 3), which are known for the occurrence of infections caused by Dobrava-Belgrade virus, the second pathogenic hantavirus in Germany, carried by the striped field mouse, Apodemus agrarius [6].

Most cases were residents of rural areas known to be endemic for Puumala virus infections, e.g. the Swabian Alb and bordering regions, Lower Franconia, the Bavarian Forest, as well as the Münster and Osnabrück regions. However, in Baden-Württemberg, the percentage of infections having occurred among residents of urban counties rose from $6.5 \%$ of the total case load

\section{FIGURE 3}

Cumulative incidence of hantavirus infections by county, Germany, January - April 2010 (n=396) and known endemic areas

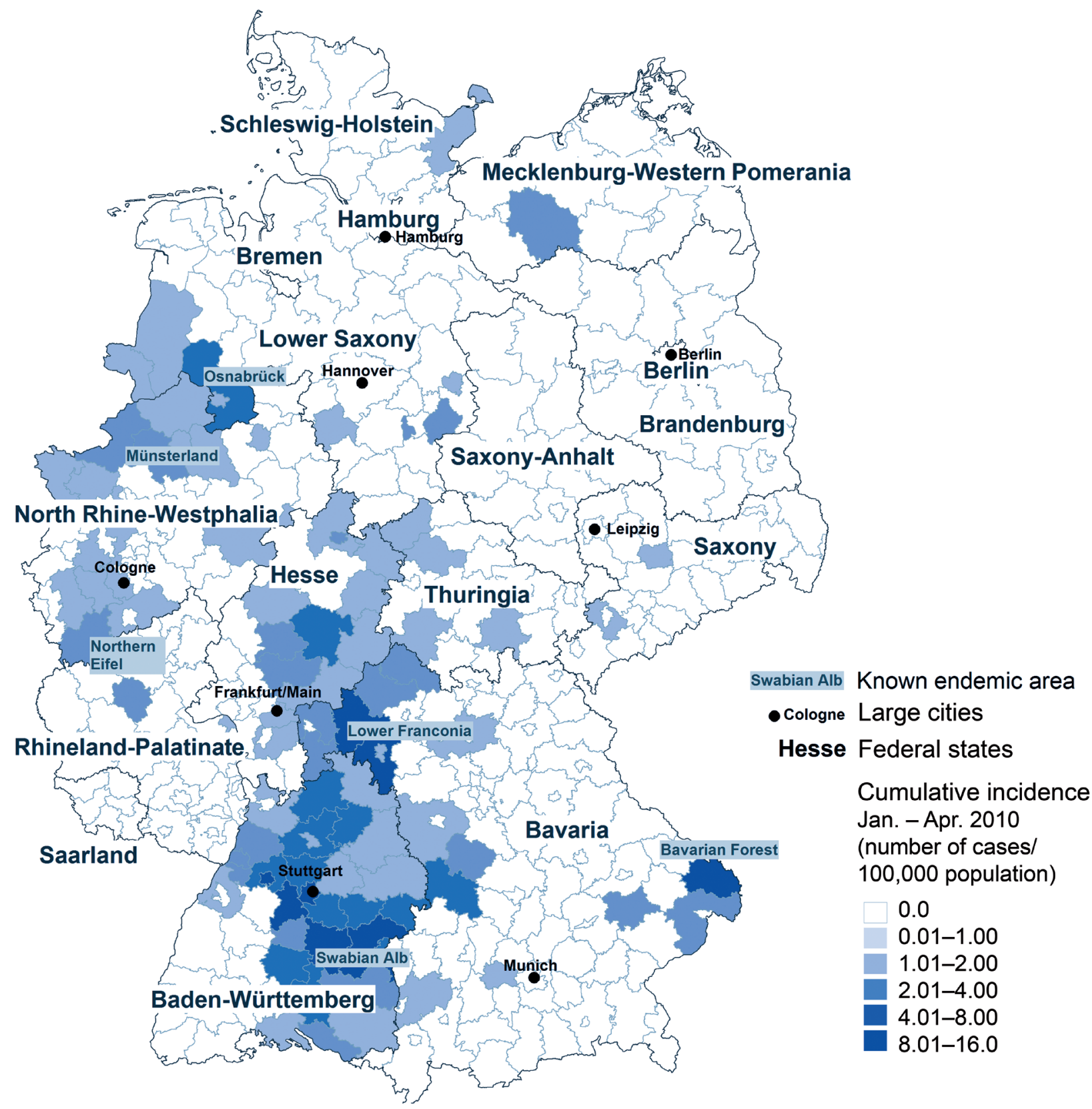


in January to April 2007 to $25.1 \%$ in the same months of 2010.

\section{Discussion}

The high number of hantavirus infections notified in early 2010, the steep increase in April and the early rise of monthly case numbers at the end of 2009 indicate that the case burden of $\mathbf{2 0 1 0}$ may exceed figures for 2007. Monthly case numbers also began to rise in the autumn of 2006, peaking at 439 infections notified in June 2007 , with a total of 1,688 cases in 2007 . On the basis of data from serological investigations and the geographical distribution of cases, the elevated number of cases is likely to be caused by infections with Puumala virus rather than Dobrava-Belgrade virus. Case numbers presented for April 2010 should be regarded as a conservative estimate because they do not include cases with pending quality assurance.

The high number of infections observed in urban environments requires further investigation. Hypotheses include bank voles moving close to human habitats due to an exceptionally cold and snowy winter, increasing presence of the animals in periurban areas used for human recreation (e.g. forests close to urban areas), as reported previously for Cologne in $2005[2,3]$, or more basic shifts in the epidemiology of Puumala virus in the bank vole population. The increasing incidence in older age groups cannot be fully explained by available data but is considered to be related to exposure rather than host factors.

Fluctuations in the population size of bank voles and the proportion of infected animals may be one factor explaining the sequence of years with very different numbers of human infections [7]. Several institutions in Germany currently cooperate in an effort to implement an appropriate monitoring system for the rodent reservoir to further study the correlation of host abundance, hantavirus prevalence and frequency of human infections [8]. Initial investigations conducted in selected trapping sites of endemic areas demonstrated a population density of 78 (standard deviation (SD): \pm 12 ) bank voles per hectare $\left(10,000 \mathrm{~m}^{2}\right)$ in April 2010 in North Rhine-Westphalia and 99 (SD: \pm 51 ) in BadenWürttemberg (all values are minimum number alive measured by live trapping in three woodlots per state) (J. Jacob, S. Schmidt, U. Rosenfeld, C. Imholt, R.G. Ulrich, unpublished data). Spring vole density in these states was thus close to multi-annual peak densities of 100 voles per hectare [9] and higher than measured previously at comparable sites in the month of April: 44 (SD: \pm 37 ) voles per hectare [10].

\section{Prevention}

To date, there is no WHO-approved hantavirus vaccine available [11]. Measures should therefore focus on prevention of exposure to rodents and their excreta, particularly in areas known to be endemic for hantavirus infections. This includes keeping houses and their surroundings free from bank voles and using appropriate protection (particle-filtering masks and gloves) when disposing of dead animals. When cleaning sheds, barns, attics or similar rooms where rodents might have nested, virus particles can be stirred up when sweeping or vacuuming. Therefore, surfaces should be moistened before cleaning (e.g. by spraying with a mix of water and household cleaner). The general public in endemic areas and people with an increased risk of occupational hantavirus infection (e.g. forestry workers and construction workers) should be informed about the ongoing, increased risk of infection and appropriate measures should be recommended.

We currently have only limited information on hantavirus infections in countries neighbouring Germany and would welcome feedback on this report from other institutions in Europe.

\section{Acknowledgements}

The authors would like to thank local health departments and partners in the networks "Rodent-borne pathogens" and Arbeitskreis "Mäuse im Forst" as well as additional collaborators for their support and Sabrina Schmidt, Ulrike Rosenfeld and Christian Imholt for provision of unpublished data.

\section{References}

1. Olsson GE, Dalerum F, Hornfeldt B, Elgh F, Palo TR, Juto P, et al. Human hantavirus infections, Sweden. Emerg Infect Dis. 2003.9(11):1395-401.

2. Essbauer SS, Schmidt-Chanasit J, Madeja EL, Wegener W, Friedrich R, Petraityte R, et al. Nephropathia epidemica in metropolitan area, Germany. Emerg Infect Dis. 2007;13(8):1271-3.

3. Abu Sin M, Stark K, van Treeck U, Dieckmann H, Uphoff H, Hautmann W, et al. Risk factors for hantavirus infection in Germany, 2005. Emerg Infect Dis. 2007;13(9):1364-6.

4. Winter $\mathrm{CH}$, Brockmann SO, Piechotowski I, Alpers K, an der Heiden M, Koch J, et al. Survey and case-control study during epidemics of Puumala virus infection. Epidemiol Infect. 2009;137(10):1479-85.

5. Hofmann J, Meisel H, Klempa B, Vesenbeckh SM, Beck R, Michel D, et al. Hantavirus outbreak, Germany, 2007. Emerg Infect Dis. 2008;14(5):850-2

6. Sibold C, Ulrich R, Labuda M, Lundkvist A, Martens H, Schutt $M$, et al. Dobrava hantavirus causes hemorrhagic fever with renal syndrome in central Europe and is carried by two different Apodemus mice species. J Med Virol. 2001;63(2):158-67.

7. Tersago K, Verhagen R, Servais A, Heyman P, Ducoffre G, Leirs $H$. Hantavirus disease (nephropathia epidemica) in Belgium: effects of tree seed production and climate. Epidemiol Infect. 2009;137(2):250-6.

8. Ulrich RG, Schmidt-Chanasit J, Schlegel M, Jacob J, Pelz HJ, Mertens M, et al. Network "Rodent-borne pathogens" in Germany: longitudinal studies on the geographical distribution and prevalence of hantavirus infections. Parasitol Res. 2008;103 Suppl 1:S121-9.

9. Bujalska G. Peak numbers of Clethrionomys glareolus (Schreber 1780) and the mechanisms involved. Pol Ecol Stud. 1995;21:397-411.

10. Jacob R. The response of small mammal populations to flooding. Mammalian Biol. 2003;68:102-111.

11. Maes P, Clement J, Van Ranst M. Recent approaches in hantavirus vaccine development. Expert Rev Vaccines. 2009;8(1):67-76. 